\title{
STUDY OF IRREDUCIBLE BALANCED PAIRS FOR SUBSTITUTIVE LANGUAGES
}

\author{
JULIEN BERNAT ${ }^{1}$
}

\begin{abstract}
Let $\mathcal{L}$ be a language. A balanced pair $(u, v)$ consists of two words $u$ and $v$ in $\mathcal{L}$ which have the same number of occurrences of each letter. It is irreducible if the pairs of strict prefixes of $u$ and $v$ of the same length do not form balanced pairs. In this article, we are interested in computing the set of irreducible balanced pairs on several cases of languages. We make connections with the balanced pairs algorithm and discrete geometrical constructions related to substitutive languages. We characterize substitutive languages which have infinitely many irreducible balanced pairs of a given form.
\end{abstract}

Mathematics Subject Classification. 68R15.

\section{INTRODUCTION}

This article deals with substitutions, that is, free morphisms on the monoid $\mathcal{A}^{*}$ generated by a finite set $\mathcal{A}$ with the concatenation. A substitution $\sigma$ naturally defines a symbolic dynamical system $\left(\mathcal{X}_{\sigma}, S\right)$, which may be split in topological factors. In the following, we only consider primitive substitutions; in this case, $\left(\mathcal{X}_{\sigma}, S\right)$ has a unique topological factor.

When $\sigma$ is a $d$-letter Pisot type substitution, it is possible to construct a geometrical representation of $\left(\mathcal{X}_{\sigma}, S\right)$ known as the Rauzy fractal of the substitution. The Rauzy fractal $\mathcal{T}$ is a compact subset of $\mathbb{R}^{d-1}$ which is equal to the adherence of its inner points, and which has positive Lebesgue measure [35]. Furthermore, $\left(\mathcal{X}_{\sigma}, S\right)$ has a minimal translation on the torus $\mathbb{R}^{d} / \mathcal{T}$ as a topological factor [13].

When the substitution $\sigma$ is unimodular, several combinatorial conditions known as coincidence conditions provide additional knowledge on $\left(\mathcal{X}_{\sigma}, S\right)$. Under the strong coincidence property, $\left(\mathcal{X}_{\sigma}, S\right)$ is measure-theoretically isomorphic to the

\footnotetext{
Keywords and phrases. Substitutive languages, balanced pairs, algorithm on words.

1 IML CNRS UMR 6206 Campus de Luminy, Case 90713288 Marseille, Cedex 9, France; bernat@iml .univ-mrs.fr
} 
exchange of $d$ domains defined almost everywhere on the associated Rauzy fractal [3]. When the super-coincidence property holds (see [23]), the Rauzy fractal generates a periodic tiling of the space, that is, there exists a lattice $\Lambda$ such that $\left(\mathcal{X}_{\sigma}, S\right)$ has a toral representation as a fundamental domain for $\mathbb{R}^{d-1} / \Lambda$, see [3]. Among unimodular Pisot type substitutions, the super-coincidence property is also equivalent to $\left(\mathcal{X}_{\sigma}, S\right)$ having a discrete spectrum, or being metrically isomorphic to a translation on a compact abelian group (see $[22,29,30])$. Note that the super coincidence condition implies the strong coincidence condition. At the moment, we do not know any Pisot type unimodular substitution which does not satisfy either the super-coincidence or the strong coincidence property.

We are interested in the connection between the study of coincidence conditions and combinatorial properties of $\mathcal{L}_{\sigma}$, the language generated by the substitution $\sigma$. For a language $\mathcal{L}$ and $u, v \in \mathcal{L}$, we say that the pair $(u, v)$ is balanced if:

$$
\text { for any letter } l \in \mathcal{A},|u|_{l}=|v|_{l} \text {. }
$$

Balanced pairs $(l, l)$, where $l \in \mathcal{A}$, are called trivial pairs. The notion of balanced pairs has been introduced by Queffélec in [30], following an idea of Host. Note that, by definition, $(u, v)$ cannot be a balanced pair if $|u| \neq|v|$. Hence we define the length of the balanced pair $(u, v)$ as the quantity $|u|=|v|$.

Remark 0.1. Trivial pairs are introduced in [34] as coincidences. We prefer to avoid this term, since it has an other meaning in the framework of substitutive dynamical systems.

When $(u, v)$ is a balanced pair with $|u| \geqslant 2$, we say that $(u, v)$ is an irreducible balanced pair if the pairs of strict prefixes of $u$ and $v$ are not balanced. For any balanced pair $(u, v)$, we call reduction of $(u, v)$ the set of irreducible balanced pairs $\left(u^{(i)}, v^{(i)}\right)_{i \in \llbracket 1, m \rrbracket}$ such that $u=u^{(1)} \ldots u^{(m)}$ and $v=v^{(1)} \ldots v^{(m)}$. Obviously, $(u, v)$ is irreducible if and only if $m=1$.

Remark 0.2. We consider that trivial pairs are irreducible balanced pairs as well. However, we choose the convention that $(\varepsilon, \varepsilon)$ is not an irreducible balanced pair.

Example 0.3. Let $\mathcal{A}=\{a, b\}$. The pair $(a a b, b a a)$ is irreducible. The pair (abaaba,babaaa) is reducible. The reduction of (abaaba,babaaa) is $\{(a b, b a)$, $(a a b, b a a),(a, a)\}$.

Any substitution $\sigma$ defines an action on irreducible balanced pairs. This action may be represented by the balanced pairs algorithm, detailed in Section 3. Queffélec noticed in [30] that, if the balanced pairs algorithm associated with a substitutive language terminates, and if the dominant eigenvalue of the incidence matrix of the algorithm is less than the dominant eigenvalue of the incidence matrix of $\sigma$, then $\left(\mathcal{X}_{\sigma}, S\right)$ has a (purely) discrete spectrum. More recently, it is proven in the forthcoming study [10] that, for any Pisot type substitution, the super-coincidence holds if and only if the strong coincidence holds and the balanced pairs algorithm terminates. Hence the study of irreducible balanced pairs associated with substitutive languages, and the action of $\sigma$ on these pairs, is closely 
related to the determination of combinatorial and ergodic properties for $\left(\mathcal{X}_{\sigma}, S\right)$; see also $[7,8,27,34]$.

This article is structured in the following form. In Section 1, we introduce the definitions and notation. In Section 2, we study the set of irreducible balanced pairs for Sturmian languages, and for languages associated with Arnoux-Rauzy words. We prove that the set of irreducible balanced pairs for the Fibonacci case may be explicitly computed (Cor. 2.3). Although we are not able to compute the whole set of irreducible balanced pairs for Arnoux-Rauzy words, we compute a particular class of irreducible balanced pairs with Proposition 2.5.

In Section 3, we introduce the balanced pair algorithm. We study with Proposition 3.4 the action of the balanced pair algorithm on the Fibonacci example. Finally, we establish a connection with the discrete geometrical representation of Rauzy fractals in Section 4. Notably, we characterize with Theorem 4.9 substitutive languages that have infinitely many irreducible balanced pairs. We end our study by a non-exhaustive list of open questions in Section 5 .

\section{DEFInitions}

For convenience, we denote by $\llbracket i, j \rrbracket$ the set of integers $k$ such that $i \leqslant k \leqslant j$.

\subsection{WORDS}

Let $\mathcal{A}$ be an alphabet, that is, a finite set of elements called letters. Endowed with the concatenation map, $\mathcal{A}$ generates a free monoid that is denoted by $\mathcal{A}^{*}$. We denote by $\varepsilon$ the empty word. A language $\mathcal{L}$ is a subset of $\mathcal{A}^{*}$; its elements are the finite words. Any word $u$, finite or infinite, defines a language if we consider the set of factors of $u$, that is, the blocs of consecutive letters occurring in $u$.

Let $u_{1}, \ldots, u_{n}$ be letters of $\mathcal{A}$. The integer $n$ is called length of the word $u=$ $u_{1} \ldots u_{n}$. For any $i \in \llbracket 0, n \rrbracket, \operatorname{pref}_{i}(u)=u_{1} \ldots u_{i}$ is the prefix of length $i$ of $u$. We set the center of $u$ as $\varepsilon$ if $n$ is even, or as the letter $u_{\frac{n+1}{2}}$ if $n$ is odd. The mirror image is the map: $\mathcal{A}^{*} \rightarrow \mathcal{A}^{*}, u \longmapsto \tilde{u}$, where $\tilde{u}=u_{n} \ldots u_{1}$. Fixed points for the mirror image map are called palindromes.

For any $l \in \mathcal{A}$, we denote by $|u|_{l}$ the number of occurrences of the letter $l$ in $u$. Let $k$ be a positive integer. The language $\mathcal{L}$ is said to be $k$-balanced if, for all words $u, v \in \mathcal{L}$, one has $\max _{l \in \mathcal{A}}\left\{|u|_{l}-|v|_{l}\right\} \leqslant k$. See also [1,37] for a study of balanced languages.

Remark 1.1. A language that is 1-balanced is often said balanced. We do not use this terminology, which stands in our study for a property on pairs of words.

For any positive integer $n$ and any language $\mathcal{L}$, we set $i_{\mathcal{L}}(n)$ as the number of irreducible balanced pairs in $\mathcal{L}$ of length $n$. The complexity map of the language $\mathcal{L}$ is the map $p_{\mathcal{L}}: \mathbb{N} \rightarrow \mathbb{N}, p_{\mathcal{L}}(n)$ being the number of distinct words of length $n$ in $\mathcal{L}$. The language $\mathcal{L}$ is said to be Sturmian if $\mathcal{L}$ is the set of factors of a one-sided sequence, such that $p_{\mathcal{L}}(n)=n+1$ for any positive integer $n$. 
Let $u \in \mathcal{L}$. If there exist $a, b \in \mathcal{A}$ such that $a u$ and $b u \in \mathcal{L}$ (respectively $u a$ and $u b \in \mathcal{L}), u$ is said to be a left special factor (resp. right special factor). A left (right) special factor $u$ is total if for any $a \in \mathcal{A}$, au $\in \mathcal{L}(u a \in \mathcal{L})$. A (total) bispecial factor is a word which is both (total) left special and (total) right special.

When $u$ is an infinite word, we say that $u$ is a left special factor if any prefix of $u$ is a left special factor.

An infinite word $u$ is said to be uniformly recurrent if, for any factor $w$, there exists an integer $C$ such that $w$ is a factor of any factor of length $C$ in $u$. Let $\mathcal{L}$ be the language which consists of the factors of a uniformly recurrent word $w$ defined on a $d$-letter alphabet with $d \geqslant 3$. We say that $w$ is an Arnoux-Rauzy word [4] if, for any positive integer $n$, there exist a unique left-special factor and a unique right-special factor of length $n$, which are both total. In the following, we call Arnoux-Rauzy language the language which consists of the set of factors of an Arnoux-Rauzy word. An Arnoux-Rauzy word $u$ is characteristic if the set of left special factors coincides with the set of prefixes of $u$.

It is proven in [31] that, for any Arnoux-Rauzy language and any positive integer $n$, the right-special factor of length $n$ is the mirror image of the leftspecial factor of the same length. Hence any Arnoux-Rauzy language is stable under mirror image. Moreover, as a generalization of the study on Rauzy graphs associated with Arnoux-Rauzy words on a three-letter alphabet [4], one gets the following result:

Proposition 1.2. Any Arnoux-Rauzy language has infinitely many bispecial words.

\subsection{Substitutions}

Let $\mathcal{A}$ be the $d$-letter alphabet $\left\{a_{i}\right\}_{i \in \llbracket 1, d \rrbracket}$, with $d$ an integer $\geqslant 2$. A substitution is a morphism of monoid on $\mathcal{A}^{*}$. The substitution $\sigma$ is said non-erasing if for every $a \in \mathcal{A}, \sigma(a) \neq \varepsilon$. The incidence matrix of $\sigma$ is defined as the square matrix $M_{\sigma} \in \mathcal{M}_{d}(\mathbb{N})$ such that, for every $(i, j) \in \llbracket 1, d \rrbracket^{2}, M_{\sigma}[i, j]=\left|\sigma\left(a_{j}\right)\right|_{a_{i}}$.

The substitution $\sigma$ is primitive if there exists a positive integer $n$ such that for every $(i, j) \in \llbracket 1, d \rrbracket^{2}, M_{\sigma}^{n}[i, j] \geqslant 1$. We denote by $\mathcal{L}_{\sigma}$ the set of factors of the words $\left\{\sigma^{k}(l) \mid l \in \mathcal{A}, k\right.$ positive integer $\}$. When $\sigma$ is primitive, $\mathcal{L}_{\sigma}$ is the set of factors of $\left\{\sigma^{k}(l) \mid k\right.$ positive integer $\}$ for any letter $l \in \mathcal{A}$. The substitution $\sigma$ is said to be unimodular if $\left|\operatorname{det} M_{\sigma}\right|=1$. See [30] for a study of the properties of dynamical systems generated by primitive substitutions.

The substitution $\sigma$ is said to be of Pisot type if the eigenvalues of the incidence matrix $M_{\sigma}$ satisfy the following: there exists a dominant eigenvalue $\beta>1$ such that, for every other eigenvalue $\alpha$, one has $0<|\alpha|<1$. Note that the characteristic polynomial of $M_{\sigma}$ is irreducible when $\sigma$ is of Pisot type. Let us remind that a Pisot number is an algebraic integer such that any Galois conjugate $\alpha \neq \beta$ satisfies $|\alpha|<1$. In particular, the dominant eigenvalue of $M_{\sigma}$ is a Pisot number when $\sigma$ is of Pisot type. It is proven in [5] that, for any Pisot type substitution $\sigma$, there exists a positive integer $k$ such that $\mathcal{L}_{\sigma}$ is $k$-balanced.

For any pair of words $(u, v)$, let us denote $(\sigma(u), \sigma(v))$ by $\sigma(u, v)$ for convenience. Let $\sigma$ be a substitution and $(u, v)$ be a balanced pair. Then $\sigma^{k}(u, v)$ is a balanced 
pair as well for any positive integer $k$. If there exists a positive integer $k$ such that the reduction of $\sigma^{k}(u, v)$ contains a trivial pair, one says that $(u, v)$ leads to a coincidence.

\section{IRREDUCIBLE BALANCED PAIRS ON SEVERAL EXAMPLES}

First, we study the case of Sturmian languages, and we compute the set of irreducible balanced pairs for the Fibonacci language. Then, we compute a subset of irreducible balanced pairs for a family of substitutions which generalize the Fibonacci substitution.

\subsection{Sturmian LANGUAGES}

We consider here the case of Sturmian languages. First, let us remind several well-known results concerning Sturmian sequences and their corresponding languages (see for instance Chap. 6 in [29]):

(1) any Sturmian language is closed under the mirror image map;

(2) a two-sided sequence is Sturmian if and only if it is 1-balanced and not eventually periodic.

Lemma 2.1. Let $\mathcal{L}$ be a 1-balanced language on $\{a, b\}$. Then the set of irreducible balanced pairs of length $\geqslant 2$ is the set of pairs $\{($ aub, bua $) ; u$ bispecial $\}$.

Proof. Let $(u, v)$ be an irreducible balanced pair of length $\geqslant 2$. By definition, $u$ and $v$ have distincts strict prefixes and suffixes; we may additionally assume without loss of generality that $u=a u^{\prime}$ and $v=b v^{\prime}$. Let $p$ be the longest common prefix of $u^{\prime}$ and $v^{\prime}$. If $p b$ is a strict prefix of $u^{\prime}$ and $p a$ is a strict prefix of $v^{\prime}$, then $(u, v)$ is not irreducible since the balanced pair $(a p b, b p a)$ occurs in its reduction. On the other hand, if $p a$ is a prefix of $u^{\prime}$ and $p b$ is a prefix of $v^{\prime}$, then apa and $b p b$ both belong to $\mathcal{L}$, which contradicts the fact that $\mathcal{L}$ is 1-balanced. Hence $u^{\prime}=p b$ and $v^{\prime}=p a$, that is, $(u, v)=(a p b, b p a)$, and $p$ is bispecial.

Conversely, let $u$ be a bispecial word. Since $\mathcal{L}$ is 1-balanced, aua and bub cannot both belong to $\mathcal{L}$. Since $\mathcal{L}$ is extendable, aub and bua $\in \mathcal{L}$. Hence $(a u b, b u a)$ is an irreducible balanced pair, which ends the proof.

Since Sturmian languages have a unique bispecial word of a given length, we obtain the following corollary, where $i(n)$ denotes the number of irreducible balanced pairs of length $n$.

Corollary 2.2. Let $\mathcal{L}$ be a Sturmian language. Then one has $i(n) \in\{0,1\}$ for any $n \geqslant 2$.

Sturmian languages may be generated by substitutions. This is for instance the case of the Fibonacci substitution $\sigma$, defined on the two-letter alphabet $\{a, b\}$ as $\sigma(a)=a b$ and $\sigma(b)=a$. The Fibonacci substitution has a unique right-sided fixed point, that we denote by $\omega$, known as the Fibonacci word. In particular, 
$\omega$ is a characteristic Sturmian word. See also [11,15-17,26,29] for further details concerning the Fibonacci substitution and Sturmian sequences.

Corollary 2.3. The irreducible balanced pairs of the Fibonacci languages are the pairs of words $\left(a \omega^{(n)} b, b \omega^{(n)} a\right)$, where $\left(\omega^{(n)}\right)_{n \in \mathbb{N}}$ is the sequence of prefixes of $\omega$ that are palindromes.

Proof. In a Sturmian language, bispecial words are exactly left special factors that are palindromes. Since the Fibonacci word $\omega$ is characteristic, bispecial words are exactly the palindromes that are prefixes of $\omega$. Hence, due to Lemma 2.1, any irreducible balanced pair is of the form $\left(a \omega^{(n)} b, b \omega^{(n)} a\right)$.

\subsection{Arnoux-Rauzy words and COnfluent Parry unit substitutions}

As Arnoux-Rauzy words generalize in some sense Sturmian sequences, we are interested in determining which properties satisfied by Sturmian languages still hold for Arnoux-Rauzy languages (see for instance [24]). Note that, due to [14], there exist Arnoux-Rauzy words that are not $k$-balanced for any positive integer $k$, which points out that Arnoux-Rauzy languages and Sturmian languages may have significant differences.

Parry numbers, introduced in $[28,32]$, are numbers $\beta>1$ for which the $T_{\beta}$-orbit of 1 is finite, where $T_{\beta}$ is the map:

$$
T_{\beta}:[0,1] \rightarrow[0,1], x \longmapsto \beta x \bmod 1 .
$$

When the $T_{\beta}$-orbit of 1 ends in $0, \beta$ is said to be a simple Parry number, a nonsimple Parry number otherwise.

A particular class of Parry numbers is the set of confluent Parry numbers. They are the positive roots of polynomials $X^{d}-\sum_{i=1}^{d-1} k X^{i}-l$, where the positive integers $k, l$ and $d$ satisfy $k \geqslant l$ and $d \geqslant 2$. Confluent Parry numbers are introduced in [21] and mainly studied in $[2,9]$.

It is possible to define for any Parry number an associated substitution [20, 36], which has a unique right-sided fixed point $\omega$. This property implies that the strong coincidence condition is satisfied. In the particular case of confluent Parry numbers, we obtain the class of confluent Parry substitutions, which are substitutions $\sigma$ defined on the $d$-letter alphabet $\left\{a_{i}\right\}_{i \in \llbracket 1, d \rrbracket}$ by:

$$
\sigma\left(a_{i}\right)=a_{1}^{k} a_{i+1} \text { if } i<d \text { and } \sigma\left(a_{d}\right)=a_{1}^{l} .
$$

Among the class of Parry substitutions, confluent Parry substitutions are exactly those for which any of the following equivalent properties hold [2]:

$\omega$ has infinitely many prefixes that are palindromes,

$\mathcal{L}_{\sigma}$ is stable under mirror image. 
Moreover, due to [2], confluent Parry unit substitutions (for which $l=1$ ) define characteristic Arnoux-Rauzy sequences.

Example 2.4. The 3-letter substitution $\sigma$ defined as $\sigma(a)=a b, \sigma(b)=a c, \sigma(c)=$ $a$, known as the Tribonacci substitution, is a confluent Parry unit substitution, with $k=1$ and $d=3$.

The following proposition provides a partial generalization of Corollary 2.3.

Proposition 2.5. Let $\mathcal{L}$ be an Arnoux-Rauzy language. There are infinitely many irreducible balanced pairs of the form $\left(l u l^{\prime}, l^{\prime} u l\right)$, where $u$ is a total bispecial factor and $l, l^{\prime}$ are distinct letters.

Proof. Let $\mathcal{L}$ be an Arnoux-Rauzy language. Due to Proposition 1.2, there exist infinitely many bispecial words $\left(u^{(n)}\right)_{n \in \mathbb{N}}$, and these words are palindromes. Hence there exist distinct letters $l, l^{\prime}$ such that $l^{\prime} u^{(n)} l \in \mathcal{L}$. Since $u^{(n)}$ is a palindrome, and since $\mathcal{L}$ is stable under mirror image, one has $l u^{(n)} l^{\prime} \in \mathcal{L}$. As a consequence, $\left(l u^{(n)} l^{\prime}, l^{\prime} u^{(n)} l\right)$ is an irreducible balanced pair.

Remark 2.6. The irreducible balanced pairs computed in Corollary 2.3 and Proposition 2.5 are of the form $(a u b, b u a)$, and of the form $(u, \widetilde{u})$ as well. However, there may exist irreducible balanced pairs that are not of these forms in the case of Arnoux-Rauzy languages. For instance, (cababa,baabac) is an irreducible balanced pair for the language generated by the Tribonacci substitution.

Note that there does not exist an irreducible balanced pair of any length. For instance, the Tribonacci language does not contain any irreducible balanced pair of length 8 .

\section{BALANCED PAIRS ALGORITHM}

First, we introduce in Section 3.1 the balanced pairs algorithm. Then, we study in Section 3.2 the action of the balanced pairs algorithm for the Fibonacci language.

\subsection{DeFinition OF THE BALANCED PAIRS ALGORITHM}

Let us recall the notion of return word. For any recurrent infinite word $u$ and any factor $w$, a return word $v$ is a finite word such that $v w$ is a factor of $u, w$ is a prefix of $v$ and $w$ occurs exactly twice in $v w$. In particular, if there exists $C>0$ such that, for any factor $w$ of $u$, any return word $v$ satisfies $|v|<C|w|$, the language is said linearly recurrent. Durand has proven in [18] that primitive substitutions generate linearly recurrent languages.

Let $\sigma$ be a primitive substitution. Let $\omega$ be a right-sided $\sigma$-periodic point, and $w$ be a non-empty prefix of $\omega$. Since $\sigma$ is primitive, there exist an integer $n$ and a finite set of return words $\left(X_{i}\right)_{i \leqslant N}$ for $w$ (see [18]). Each pair $\left(w X_{i}, X_{i} w\right)$ splits in finitely many irreducible balanced pairs. Hence $I_{1}(w)$, the set of irreducible balanced pairs obtained when reducing $\left(S^{|w|} \omega, \omega\right)$, is finite. Then, one defines by 
recurrence the set $I_{n+1}(w)$ as the set of irreducible balanced pairs occurring in the reduction of $(\sigma(u), \sigma(v))$ for all irreducible balanced pairs $(u, v)$ of $I_{n}(w)$. Let $I(w)=\bigcup_{n \geqslant 1} I_{n}(w)$. When $I(w)$ is finite, it is said that the balanced pair algorithm associated with the prefix $w$, denoted by $A(w)$, terminates.

Example 3.1. Let $\sigma$ be the Fibonacci substitution and $w=a$. Return words for $a$ are $a$ and $a b$. One has $I_{1}(a)=\{(a, a),(a b, b a)\}, I_{2}(a)=\{(a, a),(a b, b a),(b, b)\}$ and $I_{3}(a)=I_{2}(a)$. Hence $I(a)=I_{2}(a)$ and $A(a)$ terminates.

Let us study more closely the following example, introduced by Martensen as Example 3.2 in [27]. Let $\sigma$ be defined on the two-letter alphabet $\{a, b\}$ by $\sigma(a)=a a b, \sigma(b)=a b b$. The words $\sigma(a)$ and $\sigma(b)$ both have $a$ as a prefix and $b$ as a suffix. Let $\left(u_{n}\right)_{n \in \mathbb{N}}$ and $\left(v_{n}\right)_{n \in \mathbb{N}}$ be the sequences defined by $u_{0}=a b, v_{0}=b a$, such that for any integer $n \geqslant 0, a u_{n+1} b=\sigma\left(u_{n}\right)$ and $a v_{n+1} b=\sigma\left(v_{n}\right)$. Note that, by definition of $\sigma$, for any $w=w_{1} \ldots w_{n}$, one has $\sigma(w)=w^{\prime}$, where $\left|w^{\prime}\right|=3 n$ with $w_{i}^{\prime}=a$ if $i=1 \bmod 3, w_{i}^{\prime}=b$ if $i=0 \bmod 3$ and $w_{i}^{\prime}=w_{\frac{i+1}{3}}$ if $i=2 \bmod 3$.

Let $(u, v)$ be an irreducible balanced pair. Assume that $\left|\operatorname{pref}_{k}(u)\right|_{a}>\left|\operatorname{pref}_{k}(v)\right|_{a}$ for all $k \in \llbracket 1,|u|-1 \rrbracket$. Then, by definition of $\sigma$, one has $\left|\operatorname{pref}_{k}(\sigma(u))\right|_{a}>$ $\left|\operatorname{pref}_{k}(\sigma(v))\right|_{a}$ for any $k \in \llbracket 2,|\sigma(u)|-2 \rrbracket$. As a consequence, for any integer $n \geqslant 0$, $\left(u_{n}, v_{n}\right)$ is an irreducible balanced pair, and the reduction of $\left(\sigma\left(u_{n}\right), \sigma\left(v_{n}\right)\right)$ is exactly $\left\{(a, a),\left(u_{n+1}, v_{n+1}\right),(b, b)\right\}$. We deduce that, if the positive integers $i$ and $k$ are such that $\left(u_{i}, v_{i}\right)$ occurs in $I_{k}(w)$, then, for any positive integer $n,\left(u_{i+n}, v_{i+n}\right)$ occurs in $I_{k+n}(w)$. Hence, for any prefix $w \neq \varepsilon$ of $\omega, A(w)$ does not terminate.

Remark 3.2. The balanced pairs algorithm may be represented by an automaton $G$, where the states are irreducible balanced pairs, and for which we set an edge $(u, v) \rightarrow\left(u^{\prime}, v^{\prime}\right)$ whenever $\left(u^{\prime}, v^{\prime}\right)$ occurs in the reduction of $\sigma(u, v)$. On all known examples of Pisot type substitutions, and for any prefix $w$ of a $\sigma$-periodic point, the number of states in $G$ is finite; moreover $G$ has a unique strongly connected component with a number of states uniformly bounded in $w$.

The balanced pairs algorithm provides an algorithmic criterion which determines, for a given Pisot type substitution, whether the spectrum of the associated dynamical system $\left(\mathcal{X}_{\sigma}, S\right)$ is discrete, or equivalently whether the Rauzy fractal of the substitution generates a periodic tiling of the space. More precisely, one has the following result initially obtained by Livshits.

Theorem $3.3[25,34]$. Let $\sigma$ be a primitive substitution such that $\operatorname{pref}_{1}(\sigma(a))=a$. Let $\omega=\sigma^{\infty}(a)$.

(1) If there exists a positive integer $i$ such that $A\left(\operatorname{pref}_{i}(\omega)\right)$ terminates, and such that any balanced pair in $I\left(\operatorname{pref}_{i}(\omega)\right)$ leads to a coincidence, then the spectrum of $\left(\mathcal{X}_{\sigma}, S\right)$ is discrete.

(2) If there exists a positive integer $i$ such that $\operatorname{pref}_{i+1}(\omega)=\operatorname{pref}_{i}(\omega) a$, and if the spectrum of $\left(\mathcal{X}_{\sigma}, S\right)$ is discrete, then any balanced pair in $I\left(\operatorname{pref}_{i}(\omega)\right)$ leads to a coincidence. 


$$
\begin{aligned}
& u_{i}=a, v_{i}=a \quad u_{i}=a, v_{i}=a \\
& \quad\left(\begin{array}{c}
\varepsilon \\
\varepsilon
\end{array}\right) \underset{u_{i}=a, v_{i}=b}{\stackrel{u_{i}=b, v_{i}=a}{\rightleftarrows}}\left(\begin{array}{c}
b \\
\varepsilon
\end{array}\right) \underset{u_{i}=a, v_{i}=a}{\stackrel{u_{i}=b, v_{i}=b}{\rightleftarrows}}\left(\begin{array}{c}
b a \\
a
\end{array}\right)
\end{aligned}
$$

Figure 1. Potential balanced pairs produced by the action of $\sigma$ on $(u, v)$ for the Fibonacci case.

\subsection{ACtion of the BALANCED PAIRS ALGORITHM ON THE IRREDUCIBLE BALANCED PAIRS OF FIBONACCI}

We prove here with Proposition 3.4 that, for the Fibonacci case, for any positive integer $i$, the set $I\left(\operatorname{pref}_{i}(\omega)\right)$ may contain at most 4 elements which are $(a, a),(b, b),(a b, b a)$ and $(a a b, b a a)$. As a consequence, the balanced pairs algorithm $A(w)$ terminates in the Fibonacci case for any non-empty prefix $w$ of $\omega$.

As we have seen in Corollary 2.3, the irreducible balanced pairs for the Fibonacci language are of the form $\left(a \omega^{(n)} b, b \omega^{(n)} a\right)$. The following Proposition describes the action of the Fibonacci substitution $\sigma$ on the set of irreducible balanced pairs, where $\left(F_{n}\right)_{n \in \mathbb{Z}}$ denotes the Fibonacci sequence defined by $F_{n+2}=F_{n+1}+F_{n}$ for any integer $n$ with $F_{0}=F_{1}=1$.

Proposition 3.4. Let $n$ be a positive integer. The reduction of $\sigma\left(a \omega^{(n)} b, b \omega^{(n)} a\right)$ splits in:

(1) one occurrence of the trivial pair $(a, a)$,

(2) $F_{n}+1$ occurrences of the irreducible balanced pairs $(a b, b a)$,

(3) $F_{n+1}-1$ occurrences of the irreducible balanced pairs (aab, baa).

The more general case of the computation of the reduction of a balanced pair $(u, v)$ is depicted in Figure 1. Let us introduce the following definition, which is useful for the proof of the proposition.

Definition 3.5. Let $u$ and $v$ be two finite words with $v \neq \varepsilon$. The pair $(v u, u)$ is a potential balanced pair if there exists a finite word $w$ such that (vuw, uwv) is a balanced pair.

Note that, when $(u, v)$ is a potential balanced pair, then $(\sigma(u), \sigma(v))$ can be reduced in a concatenation of irreducible balanced pairs and an ending potential balanced pair.

Proof. The image of $(u, v)=\left(a \omega^{(n)} b, b \omega^{(n)} a\right)$ under $\sigma$ is $\left(a b \sigma\left(\omega^{(n)}\right) a, a \sigma\left(\omega^{(n)}\right) a b\right)$. Note that, since $\omega^{(n)}$ is both bispecial and a palindrome, it has $a$ as a prefix and as a suffix. Since $u_{1}=a$ and $v_{1}=b, \sigma\left(u_{1}, v_{1}\right)$ splits in $(a, a)$ and the potential balanced pair $(b, \varepsilon)$. For any $i \in \llbracket 1,\left|\omega^{(n)}\right| \rrbracket$, let $l_{i}$ denote the ith letter of $\omega^{(n)}$. First, suppose that $i \neq\left|\omega^{(n)}\right|$.

If $l_{i}=a$, and if the reduction of $\sigma\left(a l_{1} \ldots l_{i-1}, b l_{1} \ldots l_{i-1}\right)$ provides the potential balanced pair $(b, \varepsilon)$, then the reduction of $\sigma\left(a l_{1} \ldots l_{i}, b l_{1} \ldots l_{i}\right)$ provides the potential balanced pair $(b a b, a b)$, which splits in $(b a, a b)$ and $(b, \varepsilon)$. 
If $l_{i}=b$, and if the reduction of $\sigma\left(a l_{1} \ldots l_{i-1}, b l_{1} \ldots l_{i-1}\right)$ provides the potential balanced pair $(b, \varepsilon)$, then the reduction of $\sigma\left(a l_{1} \ldots l_{i}, b l_{1} \ldots l_{i}\right)$ provides the potential balanced pair $(b a, a)$. Since $b b$ is not a word in the Fibonacci language, one has $l_{i+1}=a$, and the reduction of $\sigma\left(a l_{1} \ldots l_{i+1}, b l_{1} \ldots l_{i+1}\right)$ provides the potential balanced pair (baab, $a a b)$, which splits in $(b a a, a a b)$ and the potential balanced pair $(b, \varepsilon)$.

If $i=\left|\omega^{(n)}\right|$, then $l_{i}=a$ and the potential balanced pair $(b, \varepsilon)$ obtained when reducing $\sigma\left(a l_{1} \ldots l_{i-1}, b l_{1} \ldots l_{i-1}\right)$ is completed as $(b a, a b)$. Hence the reduction of $\sigma\left(a \omega^{(n)} b, b \omega^{(n)} a\right)$ splits in one occurrence of the trivial pair $(a, a)$, $1+\left|\omega^{(n)}\right|_{a}-\left|\omega^{(n)}\right|_{b}$ irreducible balanced pairs of the form $(a b, b a)$ and $\left|\omega^{(n)}\right|_{b}$ irreducible balanced pairs of the form $(a a b, b a a)$.

Finally, for any positive integer $n$, one has $\omega^{(n+1)}=\sigma\left(\omega^{(n)}\right) a$, hence $\left|\omega^{(n+1)}\right|_{a}=$ $\left|\omega^{(n)}\right|_{a}+\left|\omega^{(n+1)}\right|_{b}+1$ and $\left|\omega^{(n+1)}\right|_{b}=\left|\omega^{(n)}\right|_{a}$. Since $\omega^{(0)}=\varepsilon$ and $\omega^{(1)}=a$, we obtain that, for any positive integer $n$, one has $\left|\omega^{(n)}\right|_{a}=F_{n+2}-1$ and $\left|\omega^{(n)}\right|_{b}=$ $F_{n+1}-1$. Hence $\left|\omega^{(n)}\right|_{a}-\left|\omega^{(n)}\right|_{b}+1=F_{n+2}-F_{n+1}+1=F_{n}+1$, which ends the proof.

As a direct consequence of Proposition 3.4, it is possible to compute all the irreducible balanced pairs produced by the algorithm $A(w)$, independently from $w$.

Corollary 3.6. For any prefix $w$ of $\omega$, the algorithm $A(w)$ terminates, and one has $I(w) \subset\{(a, a),(b, b),(a b, b a),(a a b, b a a)\}$.

\section{IRREDUCIBLE BALANCED PAIRS AND DISCRETE GEOMETRY}

Up to now, we have only characterized irreducible balanced pairs of the form $\left(l u l^{\prime}, l^{\prime} u l\right)$ or $(u, \tilde{u})$, and we have seen that, except for the Sturmian case, there may exist other kind of irreducible balanced pairs. In this section, we are interested in irreducible balanced pairs of the form $\left(u, S_{c}^{k}(u)\right)$, where $S_{c}$ denotes the circular shift map, defined as the map $\mathcal{A}^{*} \rightarrow \mathcal{A}^{*}$ such that $S_{c}\left(u_{1} \ldots u_{n}\right)=u_{2} \ldots u_{n} u_{1}$.

We provide in Section 4.1 a discrete geometrical construction inspired by $[12,33]$, strongly connected to the representation of numeration systems and fractal geometry (see Chap. 7 in [26] and its bibliography). We explain how this construction is related to the study of irreducible balanced pairs. Then, we characterize in Section 4.2 with Theorem 4.9 substitutive languages that have infinitely many irreducible balanced pairs of the form $\left(u, S_{c}^{k}(u)\right)$.

\subsection{Definition}

Let $\left\{\overrightarrow{v_{i}}\right\}_{i \in \llbracket 1, d \rrbracket}$ be $d$ vectors in $\mathbb{Z}^{d-1}$. We say that $\left\{\vec{v}_{i}\right\}_{i \in \llbracket 1, d \rrbracket}$ is $(d-1)$ independent if any collection of $d-1$ vectors is linearly independent. In this case, $\Lambda=\sum_{i=2}^{d} \mathbb{Z}\left(\overrightarrow{v_{i}}-\overrightarrow{v_{1}}\right)$ is a lattice of rank $d-1$, and there exists a fundamental domain for $\mathbb{Z}^{d-1} / \Lambda$ which may be seen as the union of unit hypercubes, not 


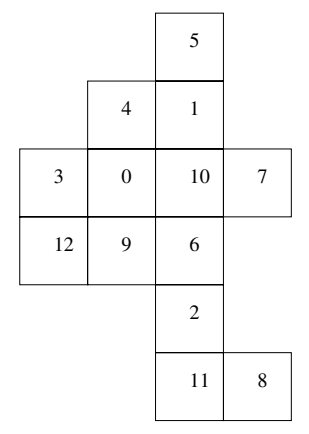

FiguRE 2. abcaababcabca is a roundwalk of $\mathcal{C}$.

necessarily connected, in $\mathbb{R}^{d-1}$. Let us see how codings are naturally defined on fundamental domains.

Let $u$ be a word on $\left\{a_{i}\right\}_{i \in \llbracket 1, d \rrbracket}$. Let $\mathcal{C}$ be a collection of unit cubes in $\mathbb{R}^{d-1}$. We say that $u$ is a roundwalk of $\mathcal{C}$, with vectors $\left\{\overrightarrow{v_{i}}\right\}_{i \in \llbracket 1, d \rrbracket}$, if the following relation holds:

$$
\sum_{i=1}^{d} \overrightarrow{v_{i}}|u|_{a_{i}}=0
$$

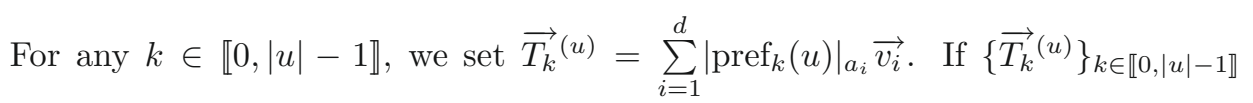
consists of $|u|$ distinct elements, $u$ is said to be an irreducible roundwalk of $\mathcal{C}$. Say differently, $u$ is an irreducible roundwalk if, for all prefixes $p, p^{\prime}$ of $u$ such that $0 \leqslant|p|<\left|p^{\prime}\right|<|u|$, one has $\sum_{i=1}^{d} \overrightarrow{v_{i}}|p|_{a_{i}} \neq \sum_{i=1}^{d} \overrightarrow{v_{i}}\left|p^{\prime}\right|_{a_{i}}$.

Remark 4.1. The irreducible roundwalk $u$ of $\mathcal{C}$ enables a labeling of the elements of $\mathcal{C}=\underset{k \in \llbracket 0,|u|-1 \rrbracket}{\cup}\left\{c_{k}\right\}$, with $c_{k}={\overrightarrow{T_{k}}}^{(u)}(0)$.

Example 4.2. There exists $\mathcal{C} \subset \mathbb{R}^{2}$ such that the word abcaababcabca is an irreducible roundwalk of $\mathcal{C}$, with vectors $\overrightarrow{v_{1}}=\overrightarrow{e_{1}}+\overrightarrow{e_{2}}, \overrightarrow{v_{2}}=-3 \overrightarrow{e_{2}}$ and $\overrightarrow{v_{3}}=$ $-2 \overrightarrow{e_{1}}+2 \overrightarrow{e_{2}}$. This example is depicted in Figure 2, with the corresponding labeling.

Lemma 4.3. Let $\mathcal{L}$ be a language, and $u \in \mathcal{L}$ such that the integers $\left\{|u|_{a_{i}}\right\}_{i \in \llbracket 1, d \rrbracket}$ are positive and relatively primes. There exist $d$ vectors $\left\{\overrightarrow{v_{i}}\right\}_{i \in \llbracket 1, d \rrbracket}$, which are $(d-1)$-independent, and $\mathcal{C} \subset \mathbb{R}^{d-1}$ such that $u$ is an irreducible roundwalk of $\mathcal{C}$ with vectors $\left\{\vec{v}_{i}\right\}_{i \in \llbracket 1, d \rrbracket}$.

Proof. Let $u \in \mathcal{L}$ such that $|u|_{a_{1}}, \ldots,|u|_{a_{d}}$ are positive and relatively primes. Consider the set of vectors $\left\{\overrightarrow{v_{i}}\right\}_{i \in \llbracket 1, d \rrbracket}$ defined by $\overrightarrow{v_{1}}=\sum_{i=2}^{d}|u|_{a_{i}} \overrightarrow{e_{i-1}}$ and $\overrightarrow{v_{i}}=$ $-|u|_{a_{i}} \overrightarrow{e_{1}}$ for all $i \geqslant 2$, where $\left\{\overrightarrow{e_{i}}\right\}_{i \in \llbracket 1, d-1 \rrbracket}$ is the canonical basis of $\mathbb{Z}^{d-1}$. Clearly, (4) holds, and $\left\{\overrightarrow{v_{i}}\right\}_{i \in \llbracket 1, d \rrbracket}$ is $(d-1)$-independent. Moreover, since the integers 
$\left\{|u|_{a_{i}}\right\}_{i \in \llbracket 1, d \rrbracket}$ are relatively primes, the elements of $\left\{{\overrightarrow{T_{k}}}^{(u)}\right\}_{k \in \llbracket 0,|u|-1 \rrbracket}$ are distinct, that is, $u$ is an irreducible roundwalk of $\mathcal{C}=\underset{k \in \llbracket 0,|u|-1 \rrbracket}{\cup}\left\{{\overrightarrow{T_{k}}}^{(u)}(0)\right\}$.

We remind that $S_{c}$ denotes the circular shift map, defined by $S_{c}\left(u_{1} \ldots u_{n}\right)=$ $u_{2} \ldots u_{n} u_{1}$.

Lemma 4.4. Let $u$ be an irreducible roundwalk of $\mathcal{C}$ with vectors $\left\{\overrightarrow{v_{i}}\right\}_{i \in \llbracket 1, d \rrbracket}$. Then, for any $i \in \llbracket 1,|u|-1 \rrbracket, S_{c}^{i}(u)$ is an irreducible roundwalk of $\mathcal{C}-\vec{T}_{i}(u)$.

Proof. Without loss of generality, one may assume that $i=1$. Clearly, if $u$ is an irreducible roundwalk, then $v=S_{c}(u)$ is an irreducible roundwalk as well with the same corresponding vectors. Since any strict prefix of $u$ is obtained as the concatenation of $u_{1}$ and a prefix of $v$, one has ${\overrightarrow{T_{k}}}^{(u)}={\overrightarrow{T_{k-1}}}^{(v)}+\vec{T}_{1}(u)$. As a consequence, $v$ is an irreducible roundwalk of $\mathcal{C}-\vec{T}_{1}(u)$.

The computation of the set of irreducible balanced pairs of a given language seems to be a tough problem. We are interested here in irreducible balanced pairs of the form $\left(u, S_{c}^{k}(u)\right)$. Note that $S_{c}^{k}(u)$ may not belong to $\mathcal{L}$; a sufficient condition for having balanced pairs of the form $\left(u, S_{c}^{k}(u)\right)$ is that $u u \in \mathcal{L}$.

Proposition 4.5. Let $u$ be an irreducible roundwalk of $\mathcal{C}$. Let $\vec{T} \in \mathbb{Z}^{d-1}$ be such that $\mathcal{C} \cap(\mathcal{C}+\vec{T})$ contains $n \geqslant 1$ elements. Let $I$ be the set of indices $i \in \llbracket 0,|u|-1 \rrbracket$ such that $\mathcal{C} \cap(\mathcal{C}+\vec{T})=\left\{c_{i}\right\}_{i \in I}$. Let $i_{0}=\min I$ and $j_{0}$ be such that $c_{j_{0}}+\vec{T}=c_{i_{0}}$. Then $\left(S^{i_{0}}(u), S^{j_{0}}(u)\right)$ is a balanced pair which reduces in $n$ irreducible balanced pairs.

Proof. Let $u$ be an irreducible roundwalk of $\mathcal{C}$. Let $\vec{T} \in \mathbb{Z}^{d-1}$ be such that $\mathcal{C} \cap(\mathcal{C}+\vec{T})$ contains $n \geqslant 1$ elements. Let $I$ be the set of indices $i \in \llbracket 0,|u|-1 \rrbracket$ such that $\mathcal{C} \cap(\mathcal{C}+\vec{T})=\left\{c_{i}\right\}_{i \in I}$. Let $i_{0}=\min I$ and $j_{0}$ be such that $c_{j_{0}}+\vec{T}=c_{i_{0}}$. Let $v=S_{c}^{i_{0}}(u)$ and $w=S_{c}^{j_{0}}(u)$. Clearly, $(v, w)$ is a balanced pair, since for any letter $l$, one has $|u|_{l}=|v|_{l}=|w|_{l}$. Moreover, for any $i \in \llbracket 1,|u| \rrbracket$, let $p$ and $q$ be respectively the prefixes of $v$ and $w$ of length $i$. Then $(p, q)$ is a balanced pair if and only if $c_{i_{0}}+\vec{T}_{i}^{(v)}=c_{j_{0}}+\vec{T}+\vec{T}_{i}^{(w)}$. Due to Lemma 4.4, this equality is equivalent to $c_{i_{0}+i}=\vec{T}+c_{j^{\prime}}$, with $j^{\prime}=j_{0}+i \bmod |u|$. Hence the number of reductions in $(v, w)$ is equal to the number of elements in $I$, that is, to the number of unit cubes in $\mathcal{C} \cap \vec{T}^{(u)}(\mathcal{C})$.

Example 4.6. Let us carry on Example 4.2 with $u=a b c a a b a b c a b c a$. One has $\overrightarrow{T_{2}}(\mathcal{C})=\overrightarrow{e_{1}}-2 \overrightarrow{e_{2}}$. One checks that $\mathcal{C} \cap\left(\overrightarrow{T_{2}}(\mathcal{C})\right)=\left\{c_{0}, c_{4}, c_{5}, c_{6}, c_{9}\right\}$, which means thanks to Proposition 4.5 that the reduction of (abcaababcabca, caababcabcaab) is $\{(a b c a, c a a b)(a, a)(b, b)(a b c, c a b)(a b c a, c a a b)\}$, see Figure 3.

Let $p_{5}$ and $p_{11}$ be respectively the prefixes of length 5 and 11 of $u$. One has $\overrightarrow{T_{5}}=$ $\overrightarrow{e_{1}}+2 \overrightarrow{e_{2}}$ and $\overrightarrow{T_{11}}=\overrightarrow{e_{1}}-5 \overrightarrow{e_{2}}$. One checks that $\overrightarrow{T_{5}}(\mathcal{C}) \cap \overrightarrow{T_{11}}(\mathcal{C})$ contains exactly one unit square (see Fig. 4), that is, $\left(S_{c}^{5}(u), S_{c}^{11}(u)\right)=$ (babcabcaabcaa, aabcaababcabc) is an irreducible balanced pair. 


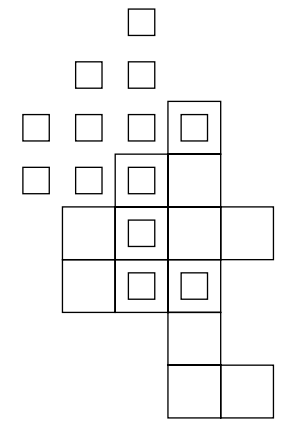

Figure 3. $\mathcal{C} \cap \vec{T}_{2}(\mathcal{C})$ contains 5 elements.

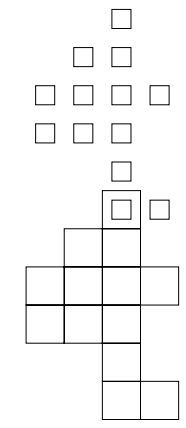

FiguRE $\quad$ 4. $\vec{T}_{5}(\mathcal{C}) \cap$ $\overrightarrow{T_{11}}(\mathcal{C})$ is a unit square.

\subsection{IRREDUCIBLE BALANCED PAIRS FOR CERTAIN SUBSTITUTIVE LANGUAGES}

Let $\sigma$ be a substitution on the alphabet $\left\{a_{i}\right\}_{i \in \llbracket 1, d \rrbracket}$. Assume that $\sigma$ satisfies the following property:

there exists $u \in \mathcal{L}$ such that $|u|_{a_{1}}, \ldots,|u|_{a_{d}}$ are relatively primes, and $u u \in \mathcal{L}_{\sigma}$.

Then, for any positive integer $k, \sigma^{k}(u u) \in \mathcal{L}_{\sigma}$. Hence, for any $i \in \llbracket 0,\left|\sigma^{k}(l)\right| \rrbracket$, $S_{c}^{i} \circ \sigma^{k}(u) \in \mathcal{L}_{\sigma}$.

Proposition 4.7. Let $\sigma$ be a unimodular primitive d-letter substitution. Let $N$ be a positive integer such that, for any $l, l^{\prime} \in \mathcal{A},\left|\sigma^{N}(l)\right|_{l^{\prime}} \geqslant 1$. Then for any integer $n \geqslant N$, there exist $(d-1)$-independent vectors $\left\{\vec{v}_{i}\right\}_{i \in \llbracket 1, d \rrbracket}$ and $\mathcal{C}_{n} \subset \mathbb{R}^{d-1}$ such that $\sigma^{n}(l)$ is an irreducible roundwalk of $\mathcal{C}_{n}$ with vectors $\left\{\overrightarrow{v_{i}}\right\}_{i \in \llbracket 1, d \rrbracket}$.

Proof. For any positive integer $n$ and any $i, j \in \llbracket 1, d \rrbracket$, the number of occurrences of the letter $a_{i}$ in $\sigma^{n}\left(a_{j}\right)$ is given by:

$$
\left|\sigma\left(a_{j}\right)\right|_{i}=M_{\sigma}^{n}[i, j] .
$$

Since $\sigma$ is unimodular, $M_{\sigma}$ is invertible. As a consequence, for any positive integer $n$, the coordinates of the first column of $M_{\sigma}^{n}$, which are $\left(\left|\sigma^{n}\left(a_{j}\right)\right|_{i}\right)_{i \in \llbracket 1, d \rrbracket}$, are relatively primes, and they are positive starting from some rank $N$ since $\sigma$ is primitive. Hence, due to Lemma 4.3, for any integer $n \geqslant N, \sigma^{n}(l)$ is an irreducible roundwalk for the set $\mathcal{C}_{n}=\underset{i \in \llbracket 0,\left|\sigma^{n}(l)\right|-1 \rrbracket}{\cup}\left\{\vec{T}_{i}^{\left(\sigma^{n}(l)\right)}(0)\right\}$.

Remark 4.8. Suppose additionally that $\sigma$ is of Pisot type; remind that, in this case, $\mathcal{H}$ is the contracting hyperspace associated with $M_{\sigma}$, and $\pi_{\mathcal{H}}$ denotes the projection on $\mathcal{H}$ along $\mathcal{D}$. Then one may define a sequence of sets $\left(\mathcal{C}_{n}\right)_{n \geqslant N}$ such that $\left(\pi_{\mathcal{H}}\left(M_{\sigma}^{-n} \mathcal{C}_{k}\right)\right)_{n \geqslant N}$ consists of uniformly bounded compact sets which tends 
for the Hausdorff metric to the Rauzy fractal of $\sigma$. See for instance the construction introduced in $[5,6]$ in the framework of generalized substitutions.

Note that Proposition 4.7 may not hold if $\sigma$ is not unimodular. For instance, let $\sigma$ be the two-letter substitution defined by $\sigma(a)=a a b$ and $\sigma(b)=a a$. Let $u=\sigma^{2}(a)=a a b a a b a a$. For any vectors $\left\{\vec{v}_{i}\right\}_{i \in \llbracket 0, k \rrbracket}$ such that (4) holds for $u$, the prefix aaba of $u$ satisfies (4) as well. Hence aabaabaa cannot be an irreducible roundwalk of any set.

Theorem 4.9. Let $\sigma$ be a unimodular primitive substitution. Suppose that there exists a word $u$ such that $u u \in \mathcal{L}$, and such that the integers $|u|_{a_{1}}, \ldots,|u|_{a_{d}}$ are relatively prime. Then $\mathcal{L}_{\sigma}$ has infinitely many irreducible balanced pairs.

Proof. Let $\sigma$ be a unimodular primitive substitution such that (5) holds. As seen in Proposition 4.7, there exist $u \in \mathcal{L}$ and a positive integer $n$ such that, for any integer $n \geqslant N, \sigma^{n}(u)$ is an irreducible roundwalk of $\mathcal{C}_{n}$ with vectors $\left\{\vec{v}_{i}\right\}_{i \in \llbracket 1, d \rrbracket}$.

Consider the labeling of $\mathcal{C}_{n}=\left\{c_{i}\right\}_{i \in \llbracket 0,\left|\sigma^{n}(u)\right|-1 \rrbracket}$ defined by the irreducible roundwalk $\sigma^{n}(u)$. Let $\vec{T}$ be a translation vector such that $\vec{T}\left(\mathcal{C}_{n}\right) \cap \mathcal{C}_{n}$ contains exactly one unit cube. Set $i, j$ such that $\vec{T}\left(\mathcal{C}_{n}\right) \cap \mathcal{C}_{n}=\left\{c_{i}\right\}=\left\{\vec{T}\left(c_{j}\right)\right\}$. Then, due to Proposition 4.5, $\left(S_{c}^{i}\left(\sigma^{n}(u)\right), S_{c}^{j}\left(\sigma^{n}(u)\right)\right)$ is an irreducible balanced pair. Since $\left(\left|\sigma^{n}(u)\right|\right)_{n \in \mathbb{N}}$ is an increasing sequence, we obtain infinitely many irreducible balanced pairs of distinct lengths.

Note that any confluent Parry unit substitution satisfies the hypothesis of Theorem 4.9 setting $u=a_{1}$.

Remark 4.10. There exist Pisot type unimodular substitutions such that (5) does not hold for any word $u$. For instance, the following Pisot type unimodular substitution, which has been computed by Pascal Ochem, generates a square-free language: $\sigma(a)=a c b a c a b c b a c b c, \sigma(b)=a c b a c a b a c b a b c, \sigma(c)=a b a c a b c b a c a b a c b c$.

\section{Open questions}

The following non-exhaustive list of questions naturally arises from the construction of geometric representations associated with a substitutive dynamical system.

When a fixed point of a primitive substitution is periodic for the shift $S$, there are finitely many irreducible balanced pairs. Is it possible to characterize other substitutive languages for which this property holds? Also, is it possible to characterize other classes of irreducible balanced pairs than those of the form $\left(u, S_{c}^{k}(u)\right)$ ?

As noticed with Remark 3.2, we do not know in the case of languages generated by Pisot type substitutions whether the balanced pair algorithm $A(w)$ may terminate for any prefix $w$ after a number of steps that does not depend on the starting irreducible balanced pairs. Say differently, does there exist a positive integer $n$ such that one has $I\left(\operatorname{pref}_{i}(\omega)\right)=I_{N}\left(\operatorname{pref}_{i}(\omega)\right)$ for any positive integer $i$ ? This question seems to be strongly related to the question of determining whether 
there exists a positive integer $n$ such that the reduction of the image under $\sigma$ of any balanced pair contains at most $N$ distinct elements.

The number of irreducible balanced pairs of length $n$ is at most $\frac{p(n)(p(n)-1)}{2}$. Since substitutive languages have a sublinear complexity map [19], $i(n)$ grows at most in $n^{2}$ up to a multiplicative constant term. However, numerical experiments suggest that this order of growth is not sharp. Is it possible to determine the exact order of growth of the number of irreducible balanced pairs of length $n$ ? For which languages is it possible to obtain the number of irreducible balanced pairs of a given length as the solution of a closed formula? Is this formula related to a rational or an algebraic function?

Acknowledgements. I am indebted to Pierre Arnoux, Valérie Berthé and the referee for their valuable comments and corrections. I also thank Julien Cassaigne and Pascal Ochem for their helpful remarks and examples.

\section{REFERENCES}

[1] B. Adamczewski, Balances for fixed points of primitive substitutions. Theoret. Comput. Sci. 307 (2003) 47-75.

[2] P. Ambrož, Z. Masáková, E. Pelantová and C. Frougny, Palindromic complexity of infinite words associated with simple Parry numbers. Ann. Inst. Fourier (Grenoble) 56 (2006) 21312160.

[3] P. Arnoux and S. Ito, Pisot substitutions and Rauzy fractals. Bull. Belg. Math. Soc. Simon Stevin 8 (2001) 181-207.

[4] P. Arnoux and G. Rauzy, Représentation géométrique de suites de complexité $2 n+1$. Bull. Soc. Math. France 119 (1991) 199-215.

[5] P. Arnoux, V. Berthé, H. Ei and S. Ito, Tilings, quasicrystals, discrete planes, generalized substitutions, and multidimensional continued fractions, in Discrete models: combinatorics, computation, and geometry (Paris, 2001). Discrete Math. Theor. Comput. Sci. Proc., AA, pp. 059-078 (electronic). Maison Inform. Math. Discrèt. (MIMD), Paris (2001).

[6] P. Arnoux, V. Berthé and S. Ito, Discrete planes, $\mathbb{Z}^{2}$-actions, Jacobi-Perron algorithm and substitutions. Ann. Inst. Fourier (Grenoble) 52 (2002) 305-349.

[7] M. Barge and B. Diamond, Proximality in Pisot Tiling Spaces. Fund. Math. 194 (2007) 191-238.

[8] M. Barge and J. Kwapisz, Geometric theory of unimodular Pisot substitutions. Amer. J. Math. 128 (2006) 1219-1282.

[9] J. Bernat, Symmetrized $\beta$-integers (2006) Submitted.

[10] J. Bernat, V. Berthé and H. Rao, On super-coincidence condition of substitutions (2006) Preprint.

[11] J. Berstel, Fibonacci words - a survey, in The Book of L, pp. 13-27. Springer-Verlag (1986).

[12] V. Berthé and R. Tijdeman, Lattices and multi-dimensional words. Theoret. Comput. Sci. 319 (2004) 177-202.

[13] V. Canterini and A. Siegel, Geometric representation of substitutions of Pisot type. Trans. Amer. Math. Soc. 353 (2001) 5121-5144.

[14] J. Cassaigne, S. Ferenczi and L.Q. Zamboni, Imbalances in Arnoux-Rauzy sequences. Ann. Inst. Fourier (Grenoble) $\mathbf{5 0}$ (2000) 1265-1276

[15] A. de Luca, A combinatorial property of the Fibonacci words. Inform. Process. Lett. 12 (1981) 193-195.

[16] X. Droubay, Palindromes in the Fibonacci word. Inform. Process. Lett. 55 (1995) 217-221. 
[17] X. Droubay, J. Justin and G. Pirillo, Epi-Sturmian words and some constructions of de Luca and Rauzy. Theoret. Comput. Sci. 255 (2001) 539-553.

[18] F. Durand, A characterization of substitutive sequences using return words. Discrete Math. 179 (1998) 89-101.

[19] F. Durand, B. Host and C. Skau, Substitutional dynamical systems, Bratteli diagrams and dimension groups. Ergod. Theor. Dyn. Syst. 19 (1999) 953-993.

[20] S. Fabre, Dépendance de systèmes de numération associés à des puissances d'un nombre de Pisot. Theoret. Comput. Sci. 158 (1996) 65-79.

[21] C. Frougny, Confluent linear numeration systems. Theoret. Comput. Sci. 106 (1992) 183219.

[22] B. Host, Valeurs propres des systèmes dynamiques définis par des substitutions de longueur variable. Ergod. Theor. Dyn. Syst. 6 (1986) 529-540.

[23] S. Ito and H. Rao, Atomic surfaces, tilings and coincidences I. Irreducible case. Israel J. Math. 153 (2006) 129-155.

[24] J. Justin and G. Pirillo, On a characteristic property of Arnoux-Rauzy sequences. RAIROTheor. Inf. Appl. 36 (2002) 385-388.

[25] A.N. Livshits, Some examples of adic transformations and automorphisms of substitutions. Selecta Math. Soviet. 11 (1992) 83-104. Selected translations.

[26] M. Lothaire, Algebraic Combinatorics On Words. Cambridge University Press (2002).

[27] B.F. Martensen, Generalized balanced pair algorithm. Topology Proc. 28 (2004) 163-178.

[28] W. Parry, On the $\beta$-expansions of real numbers. Acta Math. Acad. Sci. Hungar. 11 (1960) 401-416.

[29] N. Pytheas Fogg, Substitutions in Dynamics, Arithmetics and Combinatoric, edited by V. Berthé, S. Ferenczi, C. Mauduit and A. Siegel. Lecture Notes in Mathematics 1794 (2002).

[30] M. Queffélec, Substitution dynamical systems-spectral analysis. Lecture Notes in Mathematics 1294 (1987).

[31] R.N. Risley and L.Q. Zamboni, A generalization of Sturmian sequences: combinatorial structure and transcendence. Acta Arith. 95 (2000) 167-184.

[32] A. Rényi, Representations for real numbers and their ergodic properties. Acta Math. Acad. Sci. Hungar. 8 (1957) 477-493.

[33] S. Rosema and R. Tijdeman, The Tribonacci substitution. INTEGERS Electronic Journal of Combinatorial Number Theory 3 (2005) 1553-1732.

[34] V.F. Sirvent and B. Solomyak, Pure discrete spectrum for one-dimensional substitution systems of Pisot type. Canad. Math. Bull. 45 (2002) 697-710.

[35] V.F. Sirvent and Y. Wang, Self-affine tiling via substitution dynamical systems and Rauzy fractals. Pacific J. Math. 206 (2002) 465-485.

[36] W.P. Thurston, Groups, tilings and finite state automata. Summer 1989 AMS Colloquium Lectures (1989).

[37] L. Vuillon, Balanced words. Bull. Belg. Math. Soc. Simon Stevin 10 (2003) S787-S805.

Received January 12, 2007. Accepted November 23, 2007. 